\title{
Lipoatrophy with insulin analogues in type I diabetes
}

\author{
Amir Babiker, Vipan Datta
}

Jenny Lind Children's

Department, Norfolk and Norwich University Hospital,

Norwich, UK

\section{Correspondence to}

Dr Vipan Datta, Jenny Lind

Children's Department, Norfolk

and Norwich University

Hospital, Colney Lane,

Norwich NR4 7UY, UK;

vipan.datta@nnuh.nhs.uk

Accepted 18 May 2010

Published Online First

22 June 2010

\begin{abstract}
Lipoatrophy is a rare complication of treatment with insulin analogues. It has been reported with insulin Lispro (Eli Lilly, Indianapolis, Indiana, USA) and insulin Glargine (Sanofi-Aventis, Paris, France). To our knowledge, this is one of the first reports of lipoatrophy with Aspart, biphasic Aspart and Detemir insulin analogues (Novo Nordisk, Bagsvaerd, Denmark). We report the cases of four children with type I diabetes who were commenced on NovoMix 30 or NovoRapid/Levemir insulin injections. They developed lipoatrophy at the injection sites after 2-3 years of treatment. In two of our patients, lipoatrophy resolved when the injection sites were changed, suggesting that local factors could be the cause of lipoatrophy. However, lipoatrophy developed at the new sites in the other two patients, requiring a change of insulin preparation. Regular examination of the injection sites facilitated early detection of lipoatrophy in our patients. Lipoatrophy completely resolved over 1-2 years in all patients with no recurrence after $3-4$ years of follow-up.
\end{abstract}

\section{INTRODUCTION}

Lipoatrophy was a common cutaneous complication of insulin injections before the advent of human insulin in the 1970s and has rarely been observed since the introduction of insulin analogues. In the literature, lipoatrophy has been reported in a small number of patients, mainly with insulin Lispro (Lilly, Indianapolis, Indiana, USA) and insulin Glargine (Sanofi-Aventis, Paris, France). We report lipoatrophy in four children with type I diabetes mellitus (T1DM) who were treated with the following insulins: Aspart (NovoRapid), biphasic Aspart (NovoMix 30) and Detemir (Levemir) (Novo Nordisk, Bagsvaerd, Denmark). To our knowledge, this is one of the first reports of lipoatrophy, its management and long-term follow-up in association with Novo Nordisk insulin analogues.

\section{CASE REPORT}

Four Caucasian children with T1DM (aged 4, 5, 12 and 14 years) developed lipoatrophy (table 1). Patients 1 and 2 were commenced on NovoMix 30 insulin and patients 3 and 4 were treated with multi-dose insulin injections using NovoRapid and Glargine (patient 3) or Levemir (patient 4) insulins. The lipoatrophy was first noticed $2-3$ years after starting insulin therapy. Patients 1 and 2 developed lipoatrophy at the injection sites of NovoMix 30 (figure 1), while patient 3 developed lipoatrophy at the NovoRapid sites (figure 2) and patient 4 at the Levemir site. Glycosylated haemoglobin (HbA1c) levels at the time of developing lipoatrophy in these patients were $9.0 \%, 8.0 \%$,
$9.0 \%$ and $11.4 \%$, respectively. Insulin antibody levels were measured in three of these patients and were found to be elevated.

In patients 1 and 3 , lipoatrophy resolved when the injection site was changed. However, lipoatrophy developed at the new injection sites in patient 2 and 4 and resolved after changing the insulin preparation (NovoMix 30 to Humalog Mix 25 and Levemir to insulin Glargine). Lipoatrophy resolved over a period of $1-2$ years in all patients. HbA1c levels steadily dropped with the resolution of lipoatrophy in patients 1 and 4 but did not change in the other two children (table 1). There was no recurrence of lipoatrophy in any of our patients after 3-4 years of follow-up.

\section{DISCUSSION}

Lipoatrophy is usually a complication of T1DM treatment. Lipoatrophy was commonly seen with old insulins (bovine and porcine). It is now rarely observed since the introduction of highly purified insulins and insulin analogues.

In 2005, Ampudia-Blasco reported the case of a 39-year-old patient with T1DM who developed lipoatrophy at the injections sites with insulin Glargine. ${ }^{1}$ Most of the other previously described cases were associated with the use of insulin Lispro. A 10-year-old Caucasian girl from Yale University developed localised lipoatrophy of the subcutaneous tissue of the anterior abdominal wall a year after treatment with continuous subcutaneous insulin infusion (CSII) using Lispro insulin. ${ }^{2}$ The lipoatrophy did not progress further after switching to buffered human regular insulin using the same MiniMed CSII pump. Therefore, the authors attributed the cause to Lispro insulin. However, lipoatrophy was also reported with CSII which did not regress after changing Lispro to buffered human insulin. ${ }^{3}$ There was local hyperproduction of tumour necrosis factor $\alpha$ from macrophages leading to differentiation of adipocytes resulting in lipoatrophy. It was therefore hypothesised that Teflon cannulae used in the pump system may have induced the local immune reaction. ${ }^{3}$ Another case was reported with lipoatrophy induced by Lispro insulin but not in combination with pump therapy. ${ }^{4}$ Most recently, a 13 -year-old girl with injection-site lipoatrophy was described, also in association with Lispro insulin. ${ }^{5}$

The pathogenesis of lipoatrophy is still poorly understood. The suggested mechanisms are: repeated mechanical trauma from the injections, cryotrauma from refrigerated insulin or immune mediation. Many studies have demonstrated an association between high insulin antibodies and lipoatrophy. In a study conducted at 
Table 1 Lipoatrophy in four children with type I diabetes

\begin{tabular}{|c|c|c|c|c|}
\hline & Patient 1 & Patient 2 & Patient 3 & Patient 4 \\
\hline Age at diagnosis of T1DM (years) & 2 & 2 & 9 & 11 \\
\hline Initial insulins (indicating the insulin associated with LA) & NovoMix 30 (LA) & NovoMix 30 (LA) & NovoRapid (LA)+Glargine & NovoRapid+Levemir (LA) \\
\hline LA initially observed: age (years) & 4 & 5 & 12 & 14 \\
\hline LA initially observed: year/month & 2006/November & 2007/September & 2006/August & 2008/May \\
\hline $\mathrm{HbA} 1 \mathrm{c}$ at onset of $\mathrm{LA}(\%)$ & 9 & 8 & 9 & 11.4 \\
\hline $\mathrm{HbA} 1 \mathrm{c}$ after 3 months of resolution of LA (\%) & 7.4 & 8 & 9 & 9 \\
\hline LA resolved by changing sites & Yes & No & Yes & No \\
\hline Change of insulin analogues & No & NovoMix 30 to Humalog Mix25 & No & Levemir to Glargine \\
\hline
\end{tabular}

HbA1c, glycosylated haemoglobin; LA, lipoatrophy; T1DM, type I diabetes mellitus.

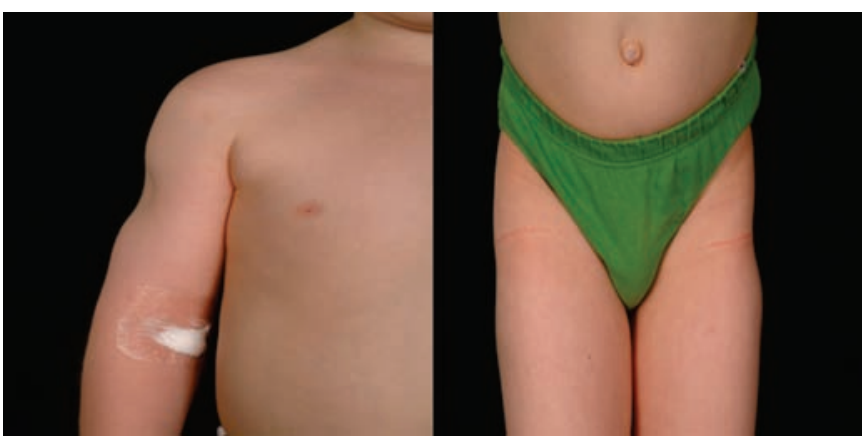

Figure 1 Lipoatrophy in the arm and leg of patient 1.

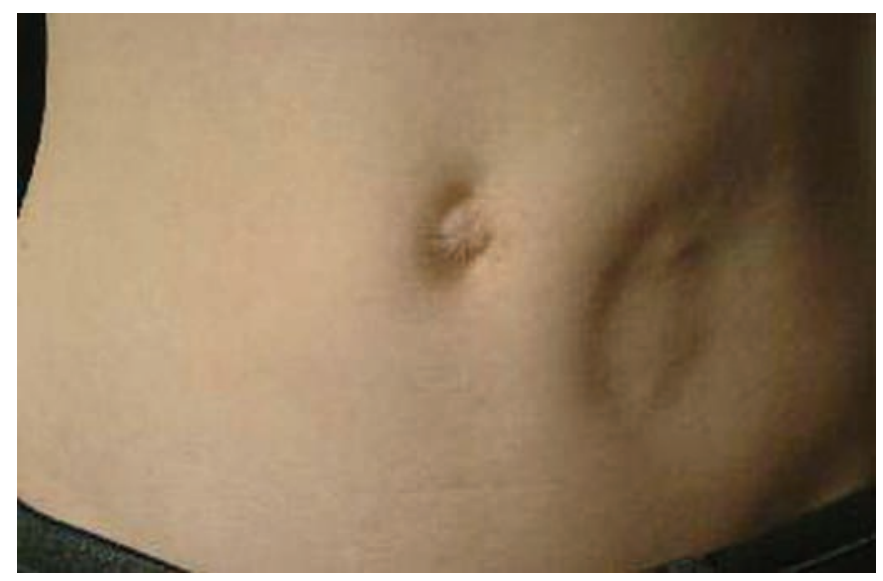

Figure 2 Lipoatrophy in the abdomen of patient 3.

the diabetes clinic of the University Children's Hospital in Munich, Germany, 112 children were randomly recruited for measurement of insulin antibodies. ${ }^{6}$ Lipoatrophy was found in four children who had significantly high insulin antibody levels. This study concluded that there is a strong association between insulin antibody levels and lipoatrophy, which implies immunity against insulin is a factor in the development of lipoatrophy. Another report in 1998 also described a significantly high insulin antibody level in an adult with diabetes. ${ }^{7}$ We observed high levels of insulin antibody in three of our cases.

The management of lipoatrophy has not yet been well established. Suggested treatments include injecting insulin around the atrophic sites in combination with dexamethasone. ${ }^{8}$ In two of our patients, lipoatrophy resolved after changing the injection sites alone, suggesting that local factors could be contributing to the development of lipoatrophy. However, in the other two children lipoatrophy developed at the new injection sites but resolved after we changed the insulin preparations. This suggests that immunity against insulin might be a contributing factor. HbA1c decreased after resolution in two of our patients but remained the same in other two. HbA1c decreased in only one of the two patients where lipoatrophy resolved after changing sites, suggesting that poor absorption from the site with lipoatrophy might be contributing to the high $\mathrm{HbA1c}$ levels in at least one of those patients. In the other two patients whose lipoatrophy resolved only after changing insulin preparation, $\mathrm{HbA1c}$ improved in only one, suggesting that immunity might have caused poor insulin absorption from the local lipoatrophy site.

\section{CONCLUSION}

Lipoatrophy is rare and can develop with all new insulin analogues. In the first instance it may be sufficient to change the injection site, but if that is not effective then changing to alternative insulin analogues may be successful, as in our experience. We recommend inspection of the injection sites at every clinic visit, which will help early detection of this rare complication of insulin treatment in children with T1DM.

Acknowledgements We would like to thank Dr NK Thalange, consultant paediatrician, for providing details of one of the patients in this case report.

Competing interests None.

Patient consent Parental consent obtained.

Provenance and peer review Not commissioned; externally peer reviewed.

\section{REFERENCES}

1. Ampudia-Blasco FJ, Girbes J, Carmena R. A case of lipoatrophy with insulin glargine: long-acting insulin analogs are not exempt from this complication. Diabetes Care 2005;28:2983.

2. Griffin ME, Feder A, Tamborlane WV. Lipoatrophy associated with lispro insulin in insulin pump therapy: an old complication, a new cause? Diabetes Care 2001;24:174

3. Ampudia-Blasco FJ, Hasbum B, Carmena R. A new case of lipoatrophy with lispro insulin in insulin pump therapy: is there any insulin preparation free of complications? Diabetes Care 2003;26:953-4.

4. Arranz A, Andia V, López-Guzmán A. A case of lipoatrophy with Lispro insulin without insulin pump therapy. Diabetes care 2004;27:625-6.

5. Sackey AH. Images in clinical medicine. Injection-site lipoatrophy. N Eng/ J Med 2009;361:e41.

6. Raile K, Noelle V, Landgraf $R$, et al. Insulin antibodies are associated with lipoatrophy but also with lipohypertrophy in children and adolescents with type 1 diabetes. Exp Clin Endocrinol Diabetes 2001;109:393-6.

7. Murao S, Hirata K, Ishida T, et al. Lipoatrophy induced by recombinant human insulin injection. Intern Med 1998;37:1031-3.

8. Kumar D, Miller L, Mehtalia S. The use of dexamethasone in treatment of lipoatrophy. Diabetes 1977;26:296-9. 